\title{
"Comparison between Propofol and Dexmedetomidine for Conscious Sedation in Patients Undergoing Outpatient Colonoscopy"
}

\author{
Sabia Siddika ${ }^{1 *}$, Syed Altaf-Uz-Zaman ${ }^{2}$, Khondaker Md. Masud ${ }^{3}$, Md. Abdul Hakim Choudhury ${ }^{4}$ \\ ${ }^{1}$ Assistant Professor \& Head, Dept. of Anesthesia, US-Bangla Medical College Hospital, Narayangonj, Bangladesh \\ ${ }^{2}$ Junior Consultant, Dept. of Anesthesia, LABAID Specialized Hospital, Dhanmondi, Dhaka, Bangladesh \\ ${ }^{3}$ Sr. Consultant, Dept. of Anesthesia, LABAID Specialized Hospital, Dhanmondi, Dhaka, Bangladesh \\ ${ }^{4}$ Assistant Professor, Dept. Of Anaesthesiology, National Institute of Kidney Diseases \& Urology (NIKDU), Dhaka, Bangladesh
}

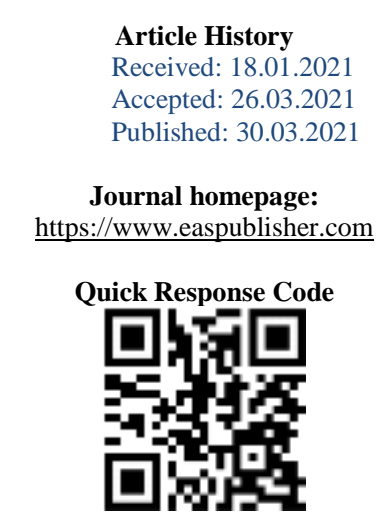

\begin{abstract}
Introduction: Colonoscopy may be a mildly painful procedure requiring conscious sedation. Though propofol may be a widely used anesthetic in day-care procedures thanks to its rapid onset and quick recovery features a drawback of requiring resuscitation maneuvers more often than the traditional methods. Dexmedetomidine, a newly introduced, highly selective $\alpha 2$-adrenergic receptor agonist possessing hypnotic, sedative, anxiolytic, sympatholytic, and analgesic properties with impressive margin of safety, must be explored to be used in conscious sedation for colonoscopy procedure among Bangladeshi population. Objective: To find out the comparison between propofol and dexmedetomidine for conscious sedation in patients undergoing outpatient colonoscopy. Materials and Methods: A prospective randomized comparative study was conducted on patients aged between 25 and 60 years with the American Society of Anesthesiologist physical status classes I and II posted for colonoscopy under monitored anesthesia Dept. LABAID Specialized Hospital, Dhanmondi, Dhaka, Bangladesh from January 2018 to December 2019. Study group was randomly divided into two groups and administered propofol and dexmedetomidine. The primary outcome variable was assessments of sedation scores between the two groups. Secondary outcome variables were pain score assessments, hemodynamic comparisons, and adverse events among the two groups. Appropriate statistical tests were applied to compare the findings. Results: After comparisons between the two groups, we found that patients on dexmedetomidine had similar sedation score as that of patients on propofol. The baseline sedation scores among the study population depicted that $>96 \%$ patients in Group 1 came under the scale of $4-5$, and in Group 2, nearly $93 \%$ patients come under scale of 4-5 which was statistically not significant $(\mathrm{P}=0.686)$. The baseline pain scale values for the patients in both the groups showed that majority in Group 1 came under the scale of 1-2 (93\%) and in scale between 1 and 2 (96\%) for Group $2(\mathrm{P}=0.059)$. However, there was a significantly higher incidence of systemic hypotension. Requirement of rescue analgesia and adverse events and other hemodynamic fluctuation were similar in both the groups. Conclusion: We conclude that dexmedetomidine has similar efficacy as propofol for conscious sedation required during colonoscopy. Occurrence of systolic hypotension was, however, significantly more among the group receiving dexmedetomidine.
\end{abstract}

Keywords: Colonoscopy, Dexmedetomidine, Propofol, Conscious Sedation, Systolic Hypotension.

Copyright () 2021 The Author(s): This is an open-access article distributed under the terms of the Creative Commons Attribution 4.0 International License (CC BY-NC 4.0) which permits unrestricted use, distribution, and reproduction in any medium for non-commercial use provided the original author and source are credited.

\section{INTRODUCTION}

Colonoscopy is a mildly painful procedure, and it requires conscious sedation. Nowadays colonoscopy is the standard procedure for diagnosis, screening, treatment and follow up for many colorectal diseases. Propofol is a widely used sedative hypnotic for day-care procedures as it is associated with faster onset and recovery of sedation [1]. It is known to cause dose-dependent respiratory depression, and this may be amplified in the presence of opioids requiring resuscitation maneuvers $[2,3]$. Dexmedetomidine is a relatively new drug approved at the end of 1999 by the Food and Drug Administration (FDA) for humans use for short-term sedation and analgesia ( $<24$ hours) in the intensive care unit (ICU). Although some patients can tolerate colonoscopy procedure without any sedation and analgesic requirements, it is a distressful procedure for most patients. Although some patients can tolerate colonoscopy procedure without any sedation and analgesic requirements, it is a distressful procedure for most patients. Earlier, various drugs such as midazolam, opioids, and ketamine have been used alone or in combination with propofol and are known to be 
associated with side effects such as respiratory depression. It possesses hypnotic, sedative, anxiolytic, sympatholytic, and analgesic properties without producing significant respiratory depression [4]. It also reduces both anesthetic and opioid analgesic requirements during the perioperative period. It has an impressive safety margin, and it may be suitable for conscious sedation during painful procedures [5]. Dexmedetomidine is a useful sedative agent with analgesic properties, hemodynamic stability and ability to recover respiratory function in mechanically ventilated patients facilitating early weaning. It was reported that dexmedetomidine provides effective analgesia and reduces postoperative morphine requirements [6]. Furthermore, combination of dexmedetomidine with fentanyl provided good pain relief during shock wave lithotripsy procedure [7]. In laparoscopic bariatric surgery, the use of dexmedetomidine significantly reduced the pain and nausea [8]. Similarly, there are plenty of studies done on the use of dexmedetomidine usefulness during procedures such as ophthalmic surgeries, gynecologic surgeries, and fiber-optic intubation [9, 13]. However, there are not many studies done on the effectiveness of dexmedetomidine use in conscious sedation during colonoscopy procedure in Bangladeshi hospital setup. Furthermore, as there is lack of literature on its effect on South Bangladeshi population, this study was conducted to compare the sedative efficacy of dexmedetomidine as compared to propofol.

\section{Materials ANd Methods}

Sixty patients aged between 25 and 60 years, the American Society of Anesthesiologist physical status classes I and II posted for colonoscopy under monitored anesthesia Dept. LABAID Specialized Hospital, Dhanmondi, Dhaka, Bangladesh from January 2018 to December 2019, were included in this study. Study participants were included according to convenience sampling method. Patients allergic to $\alpha 2-$ adrenergic agonist or sulfa drugs, history of alcohol or drug abuse, second - and third - degree heart block, cardiac, respiratory, renal, and liver diseases, pregnant women, lactating women, and patients with psychiatric disorders were excluded from this study. A written informed consent was taken from all the study participants after describing in full detail the nature and purpose of this study. Ethical clearance was obtained from the Institute's Ethics Committee.

Study participants were divided into two groups (Group 1 and Group 2) on the basis of random sampling method. Group 1 had patients receiving propofol, and Group 2 consisted of those receiving dexmedetomidine. Patients from both the groups were kept nil per oral for solids for 6 and $2 \mathrm{~h}$ for clear fluids.
All patients were given tablet ranitidine $150 \mathrm{mg}$ overnight and on the morning of the procedure. In the procedure room, electrocardiography leads that noninvasive blood pressure (BP) cuff and pulse oximeter were connected to the patients. Baseline readings of heart rate, $\mathrm{BP}$, and oxygen saturation percentage were noted. An intravenous (i.v.) access was secured using $18 \mathrm{G}$ or $20 \mathrm{G}$ cannula, and Ringer lactate/normal saline fluids were given based on the body weight of patients. All patients were premedicated with injection glycopyrrolate $0.2 \mathrm{mg}$ i.v. and analgesic injection fentanyl $0.5 \mu \mathrm{g} / \mathrm{kg}$ i.v. over $5 \mathrm{~min}$. Throughout the procedure, all patients were given a mixture of O2/N2O (4 L/4 L) as anesthetic agent using Bains circuit. A $50-\mathrm{ml}$ syringe and an electronic infusion pump were used for the study drugs throughout the procedure. Group 1 received initial loading dose of propofol 2-3 mg/kg i.v. over $10 \mathrm{~min}$, followed by a continuous i.v. infusion of $25-100 \mu \mathrm{g} / \mathrm{kg} / \mathrm{min}$ till the end of colonoscopy [14], Group 2 received initial loading dose of dexmedetomidine $1 \mu \mathrm{g} / \mathrm{kg}$ i.v over 10 $\mathrm{min}$, followed by a continuous infusion of $0.2-0.8$ $\mu \mathrm{g} / \mathrm{kg} / \mathrm{h}$, till the end of the procedure [15].

Heart rate, BP, and oxygen saturation were recorded intraoperatively at every $5 \mathrm{~min}$ interval for the first $30 \mathrm{~min}$ and subsequently at $10 \mathrm{~min}$ intervals till the end of the procedure. Patient's sedation level was assessed using modified Observer's Assessment Alertness/Sedation scale, which states Scale $5=$ responds readily to name spoken in normal tone, Scale $4=$ responds lethargically to name spoken in normal tone, Scale 3=responds after name spoken loudly, Scale $2=$ responds after mild prodding or shaking, and Scale $1=$ unarousable [16].

During the procedure, if the patient had pain after the administration of study drugs, an additional bolus dose of injection fentanyl $0.5 \mu \mathrm{g} / \mathrm{kg}$ i.v. bolus was given as a rescue analgesia. Adverse events such as hypotension (defined as systolic BP $<90 \mathrm{mmHg}$ ) were treated with fluid bolus and injection ephedrine $6 \mathrm{mg}$ i.v. bolus. Bradycardia (heart rate $<40 / \mathrm{min}$ ) was treated with injection atropine $0.6 \mathrm{mg}$ i.v. bolus. Apnea or bradypnea (respiratory rate of $<10 / \mathrm{min}$ ) was managed by manually ventilating the patient.

In the postoperative ward, all patients received oxygen using face mask at $5 \mathrm{~L} / \mathrm{min}$ for $30 \mathrm{~min}$. Heart rate, $\mathrm{BP}$, and oxygen saturation percentage were observed. Postoperative analgesia was assessed using numerical pain intensity scale at baseline and later at 30 min interval for the next $2 \mathrm{~h}$. A scale of 10 was considered as being worst possible pain and scale of 0 as no pain. Rating on the scale of $>4$ was treated with i.v. bolus of injection tramadol $1 \mathrm{mg} / \mathrm{kg}$. 


\begin{tabular}{|l|l|l|l||}
\hline Score Reactivity & Speech & Face expression & Eyes \\
\hline 5 Normal response & Normal & Normal & Clear \\
\hline $\begin{array}{l}4 \text { Lethargic response to } \\
\text { name spoken in normal } \\
\text { tone }\end{array}$ & Mild slowing & Mild relaxation & Mild ptosis \\
\hline $\begin{array}{l}\text { 3 Responds only after } \\
\text { name is called loudly or } \\
\text { repeatedly }\end{array}$ & Prominent slowing & Marked relaxation & Marked ptosis \\
\hline $\begin{array}{l}\text { 2 Responds only after mild prodding or shaking Few recognizable word 1 } \\
\text { Responds only after squeezing of the trapezius 0 Does not respond after } \\
\text { squeezing of the trapezius }\end{array}$ \\
\hline \multicolumn{3}{|c|}{ Table 1: Alertness/Sedation (OAA/S) scale. } \\
\hline
\end{tabular}

\section{Statistical Analysis}

All statistical analysis was performed using the Statistical Package for Social Science (SPSS) program, version 22 and Windows. The data were checked for normal distribution. Demographic data such as age, gender, and weight were matched using Mann-Whitney U-test. The primary outcome variable between the two groups was assessments of sedation scores. Secondary outcome variables were need for rescue analgesia, hemodynamic parameters, adverse events, and pain score assessments. Sedation score was analyzed using Fisher's exact test. Need for rescue analgesia among the two groups was analyzed by " $Z$-" test for proportions. All hemodynamic parameters were expressed as mean \pm standard deviation. Adverse events were analyzed using Fischer's exact test and Chi-square test. Pain score assessment was done by Fisher's exact test. $\mathrm{P}<0.05$ was considered as statistically significant.

\section{RESUlTS}

Table 2 shows demographic variables such as age, gender, and weight compared between the two groups. The baseline sedation scores among the study population depicted that $>96 \%$ patients in Group 1 came under the scale of 4-5, and in Group 2, nearly 93\% patients come under scale of 4-5 which was statistically not significant $(\mathrm{P}=0.686)$. The baseline pain scale values for the patients in both the groups showed that majority in Group 1 came under the scale of 1-2 (93\%) and in scale between 1 and $2(96 \%)$ for Group 2 $(P=0.059)$. Adverse events were noted among the study population during the procedure [Table 2]. Bradycardia was seen in $6.7 \%$ in Group 1 and in $16.7 \%$ in Group 2 which was not significant $(\mathrm{P}=0.424)$. Nearly $56.7 \%$ of patients in Group 2 experienced significant systolic hypotension as compared to $13.3 \%$ of patients in Group $1(\mathrm{P}=0.001)$. No significant diastolic hypotension was noted between the two groups $(P=0.256)$. The need for rescue analgesia (pain scale $>4$ ) arose in $20 \%$ of patients in Group 1, while in Group 2, this increased to $40 \%(\mathrm{P}=0.159)$. Clinically, it seemed significant though it was not significant statistically [Table 2].

Table-2: Demographic data and other characteristics. The values are expressed as mean \pm SD, numbers (N) or proportions

\begin{tabular}{|l|l|l|l|}
\hline Parameter & Group 1 & Group 2 & P \\
\hline Age $($ years $)$ & $46.17 \pm 12.6$ & $47.13 \pm 11.7$ & 0.784 \\
\hline Sex $(n)$ & & & \\
\hline Male, female & 18,12 & 22,8 & 0.273 \\
\hline Weight $(\mathrm{kg})$ & $56.37 \pm 8.0$ & $58.0 \pm 6.6$ & 0.487 \\
\hline Pain scale & & & \\
\hline Score 0 & $01(3.3 \%)$ & Nil & 0.059 \\
\hline Score 1 & $17(56.7 \%)$ & $26(86.7 \%)$ & \\
\hline Score 2 & $11(36.7 \%)$ & $03(10 \%)$ & \\
\hline Score 3 & $01(03.3 \%)$ & $01(3.3 \%)$ & \\
\hline Score 4 & Nil & Nil & \\
\hline Score $>5$ & Nil & Nil & \\
\hline Sedation score, OAA/S scale & & & \\
\hline Scale 5 & $23(76.70)$ & $24(80.0)$ & 0.686 \\
\hline Scale 4 & $06(20.0)$ & $04(13.3)$ & \\
\hline
\end{tabular}




\begin{tabular}{|l|l|l|l|}
\hline Parameter & Group 1 & Group 2 & P \\
\hline Scale 3 & $01(3.30)$ & $02(6.7)$ & \\
\hline Scale 2 & Nil & Nil & \\
\hline Scale 1 & Nil & Nil & \\
\hline Adverse events during the procedure (\%) & & & \\
\hline Bradycardia & 6.7 & 16.7 & 0.424 \\
\hline Hypotension (systolic) & 13.3 & 56.7 & $0.001^{*}$ \\
\hline Hypotension (diastolic) & 20 & 6.7 & 0.256 \\
\hline Need for rescue analgesia (\%) & $6(20 \%)$ & $12(40 \%)$ & 0.159 \\
\hline *P value $<0.05$ is considered as statistically significant. SD=Standard deviation, \\
OAA/S=Observer's Assessment Alertness/Sedation
\end{tabular}

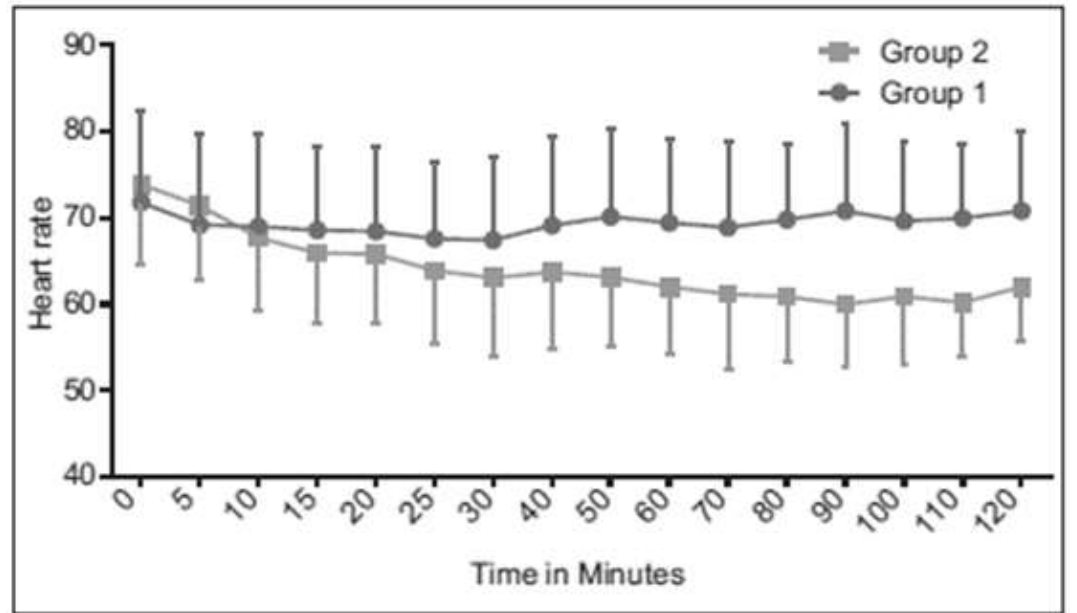

Fig-1: Hemodynamic comparisons between groups with respect to heart rate. The data are expressed as mean \pm standard deviation

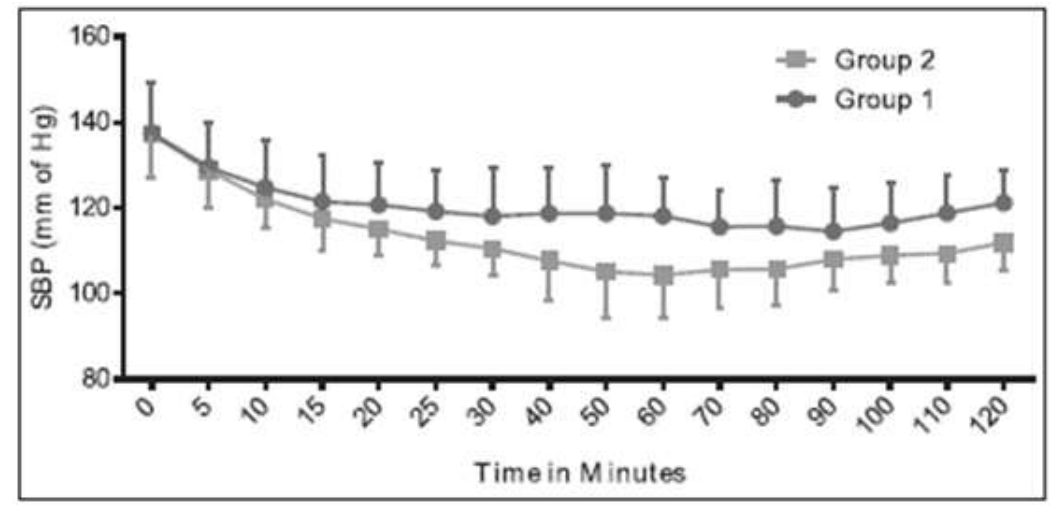

Fig-2: Hemodynamic comparisons between groups with respect to systolic blood pressure. The data are expressed as mean \pm standard deviation

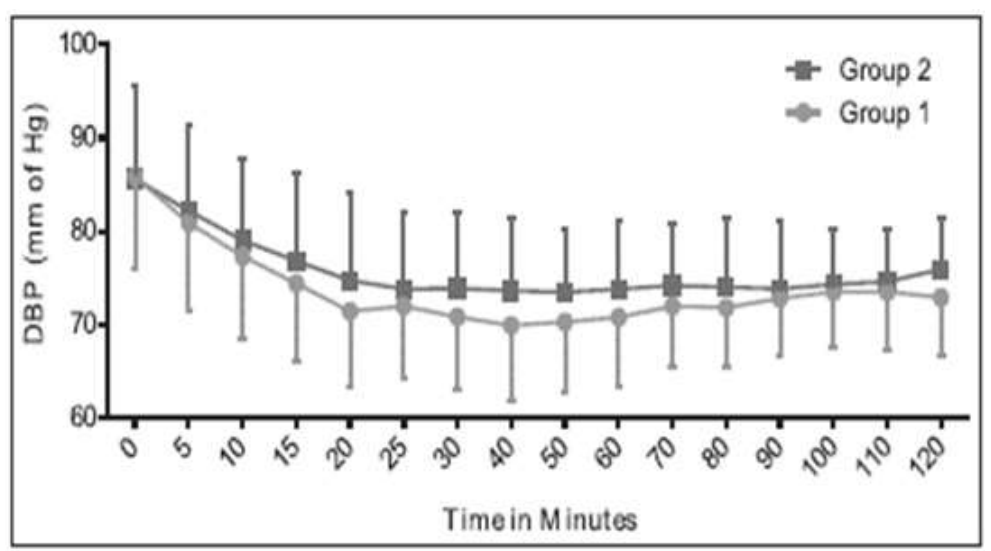

Fig-3: Hemodynamic comparisons between groups with respect to diastolic blood pressure. The data are expressed as mean \pm standard deviation 


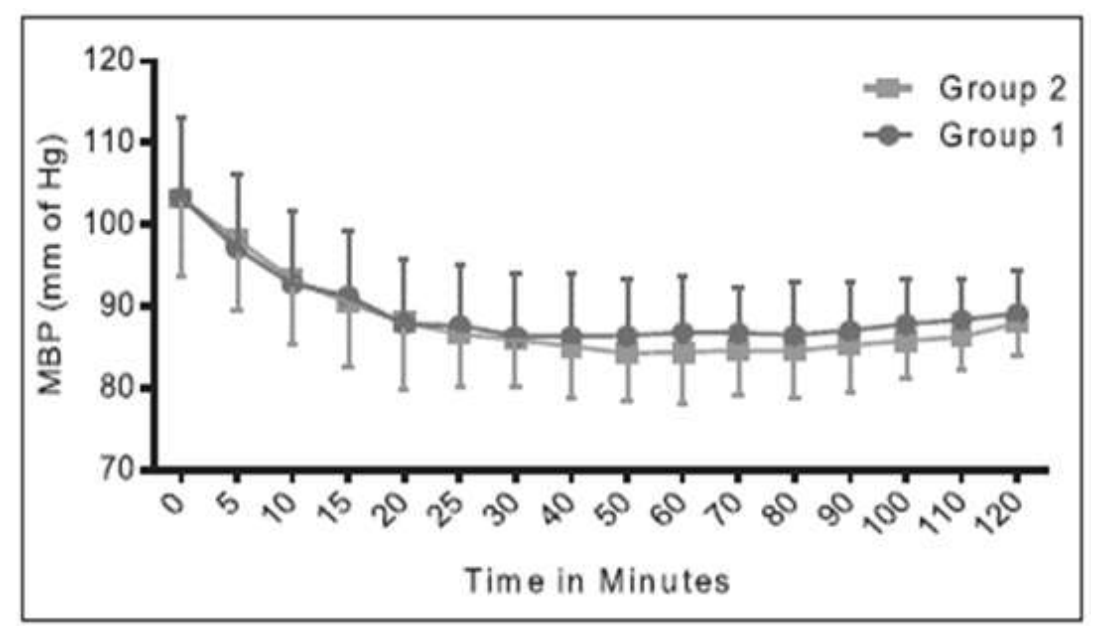

Fig-4: Hemodynamic comparisons between groups with respect to mean blood pressure. The data are expressed as mean \pm standard deviation

Figure 1 shows that when heart rate of Group 2 was compared with the baseline value, there was a decrease in the first $40 \mathrm{~min}$. In Group 1, heart rate was maintained at baseline value till the 50th min, and then, there was a surge in heart rate. Thereafter, it persisted around the same value throughout the procedure. Heart rate was comparable between the two groups. Group 2 showed an additional 15\%-20\% decline from the baseline systolic BP as compared to Group 1 [Figure 2]. Figures 3 and 4 show diastolic BP and mean arterial pressure between the two groups. Both the groups showed similar pattern with an initial fall, and thereafter, it was maintained in the same pattern.

\section{DISCUSSION}

The results of our study indicate that dexmedetomidine has similar sedation efficacy as that of propofol, a typical drug used during colonoscopy. However, patients receiving dexmedetomidine experienced a big fall in systolic BP, but it didn't amount to any end-organ damage. This finding was supported by study where they used dexmedetomidine solely for sedation [17]. Colonoscopy being a typical procedure for diagnosis, screening, treatment, and follow-up for several colorectal diseases requires conscious sedation for outpatients. Colonoscopy is usually a painful and embarrassing procedure requiring an appropriate drug for maximizing patient comfort with adequate sedation, good analgesia, and minimal adverse events [17]. Dexmedetomidine may be a potent $\alpha 2$-agonist. It decreases the central sympathetic outflow and circulating catecholamines, thus leading to systolic hypotension. Hypotension might be further exacerbated by dehydration thanks to bowel preparations and overnight fasting which is a routine protocol for colonoscopy. Studies have shown that dexmedetomidine can be used in hypertensives and coronary heart disease patients due to its hypotensive effect $[18,19]$. Other hemodynamic fluctuations such as bradycardia and diastolic hypotension were comparable to that experienced using propofol. The need for rescue analgesia was also comparable to that of propofol. The use of fentanyl $0.5 \mu \mathrm{g} / \mathrm{kg}$ i.v with dexmedetomidine achieved sufficient analgesia during the procedure. In contrast, other studies show that dexmedetomidine was combined with fentanyl $1 \mu \mathrm{g} / \mathrm{kg}$ to achieve the required analgesia. Lower dose of fentanyl was found to be sufficient among the Bangladeshi population probably due to it synergistic action with that of dexmedetomidine for sedation. Indian population is known to have a higher body fat percentage, thus increasing the sensitivity to fentanyl [20]. Furthermore, by minimizing the dose of fentanyl, opioid-induced respiratory depression can be reduced which would have otherwise negated the respiratory sparing effects of dexmedetomidine. Our findings are similar to a study which used dexmedetomidine as the sole agent and found that its use was associated with prolonged recovery, bradycardia, and hypotension [17]. We found no significant bradycardia and this can be attributed to the use of glycopyrrolate as premedication which is a vagolytic. Glycopyrrolate could have prophylactically prevented severe bradycardia or ventricular ectopics. Studies have shown that dexmedetomidine does not produce significant respiratory depression during the procedure, thus favoring its usefulness in remote locations where facilities available are limited [17, 21]. There was laboratory result that demonstrated a powerful inhibitory effect of propofol on sympathetic outflow [22]. Dexmedetomidine is also known to decrease sympathetic outflow and circulating catecholamine levels and would therefore be expected to cause the decrease of blood pressure and heart rate similar to those of propofol [22, 23]. In our study, the systolic blood pressure was decreased from baseline in both groups from the 5 th minute after starting the study drugs throughout the colonoscopy, which were explained by sympatolytic effect of both sedatives and fentanyl. However, dexmedetomidine has a direct effect at the postsynaptic vascular smooth muscles and causes vasoconstriction, and sympathoinhibitory effects are opposed. The effect of vasoconstriction appears earlier than the effect of central sympatholysis. Although there were reports that sleepiness appeared within 5 minutes 
after intravenous administration of dexmedetomidine and reached its maximum effect within 15 minutes [26], but the transient increase in blood pressure stared at one minute and peaked within 3 minutes [24]. Therefore, systolic blood pressure at the 5 th $\mathrm{min}$ in the propofol group was more decreased than in the dexmedetomidine group and the onset of hypotension was more early (10 min vs. $20 \mathrm{~min}$ ). From the figure of systolic blood pressure variables during the intraoperative period, the average blood pressure in the propofol group seem to be lower than in the dexmedetomidine group from the 5 th to 20th minute. Then after the 20th minute, the average blood pressures in both groups were similarly and the statistic showed no difference. That means the vasoconstriction effect of dexmedetomidine may be last long as 20 minutes. From subgroup analysis of the age group $>60$ years, patients in the dexmedetomidine group showed greater tendency to develop hypotension which was not different from the propofol group. This may explained by the fact that dexmedetomidine had worse effect on blood pressure in the elderly patients and should be more closely monitored for the hypotension. Propofol has limited analgesic effect and higher doses are often required, when it is used as a single agent for colonoscopy, resulting in higher sedation levels. Thus, the use of propofol in combination with other agents may be preferable to propofol alone [25]. Not documenting the recovery time and postoperative discharge time are the limitations of this study. Studying the efficacy of conscious sedation of dexmedetomidine on a larger sample and exploring the feasibility of use of this drug in procedures done in remote location form the future scope of this study.

\section{Conclusion}

In our study, dexmedetomidine has similar efficacy as propofol for conscious sedation required during colonoscopy. Need for rescue analgesia and adverse events were same as that of propofol. Occurrence of systolic hypotension was, however, significantly more among the group receiving dexmedetomidine. Hence, its usefulness on patients undergoing various procedures needs further exploration.

\section{REFERENCES}

1. Ulmer, B. J., Hansen, J. J., Overley, C. A., Symms, M. R., Chadalawada, V., Liangpunsakul, S., ... \& Rex, D. K. (2003). Propofol versus midazolam/fentanyl for outpatient colonoscopy: administration by nurses supervised by endoscopists. Clinical Gastroenterology and Hepatology, 1(6), 425-432.

2. Rex, D. K., Overley, C., Kinser, K., Coates, M., Lee, A., Goodwine, B. W., ... \& Helper, D. (2002). Safety of propofol administered by registered nurses with gastroenterologist supervision in 2000 endoscopic cases. The American journal of gastroenterology, 97(5), 1159-1163.
3. Muller, S., Borowics, S. M., Fortis, E. A., Stefani, L. C., Soares, G., Maguilnik, I., ... \& Caumo, W. (2008). Clinical efficacy of dexmedetomidine alone is less than propofol for conscious sedation during ERCP. Gastrointestinal endoscopy, 67(4), 651-659.

4. Hall, J. E., Uhrich, T. D., Barney, J. A., Arain, S. R., \& Ebert, T. J. (2000). Sedative, amnestic, and analgesic properties of small-dose dexmedetomidine infusions. Anesthesia \& Analgesia, 90(3), 699-705.

5. Dyck, J. B., Maze, M., Haack, C., Vuorilehto, L., \& Shafer, S. L. (1993). The pharmacokinetics and hemodynamic effects of intravenous and intramuscular dexmedetomidine hydrochloride in adult human volunteers. The Journal of the American Society of Anesthesiologists, 78(5), 813820.

6. Gurbet, A., Basagan-Mogol, E., Turker, G., Ugun, F., Kaya, F. N., \& Ozcan, B. (2006). Intraoperative infusion of dexmedetomidine reduces perioperative analgesic requirements. Canadian Journal of Anesthesia, 53(7), 646-652.

7. Kaygusuz, K., Gokce, G., Gursoy, S., Ayan, S., Mimaroglu, C., \& Gultekin, Y. (2008). A comparison of sedation with dexmedetomidine or propofol during shockwave lithotripsy: a randomized controlled trial. Anesthesia \& Analgesia, 106(1), 114-119.

8. Tufanogullari, B., White, P. F., Peixoto, M. P., Kianpour, D., Lacour, T., Griffin, J., ... \& Provost, D. A. (2008). Dexmedetomidine infusion during laparoscopic bariatric surgery: the effect on recovery outcome variables. Anesthesia \& Analgesia, 106(6), 1741-1748.

9. Jaakola, M. L., Ali-Melkkilä, T., Kanto, J., Kallio, A., Scheinin, H., \& Scheinin, M. (1992). Dexmedetomidine reduces intraocular pressure, intubation reponses and anaesthetic requirements in patients undergoing ophthalmic surgery. British Journal of Anaesthesia, 68(6), 570-575.

10. Ghali, A., Mahfouz, A. K., Ihanamäki, T., \& El Btarny, A. M. (2011). Dexmedetomidine versus propofol for sedation in patients undergoing vitreoretinal surgery under sub-Tenon's anesthesia. Saudi journal of anaesthesia, 5(1), 36.

11. Aho, M., Erkola, O., Kallio, A., Scheinin, H., \& Korttila, K. (1992). Dexmedetomidine infusion for maintenance of anesthesia in patients undergoing abdominal hysterectomy. Anesthesia and analgesia, 75(6), 940-946.

12. Aantaa, R., Kanto, J., Scheinin, M., Kallio, A., \& Scheinin, H. (1990). Dexmedetomidine, an a2adrenoceptor agonist, reduces anesthetic requirements for patients undergoing minor gynecologic surgery. The Journal of the American Society of Anesthesiologists, 73(2), 230-235.

13. Bergese, S. D., Bender, S. P., McSweeney, T. D., Fernandez, S., Dzwonczyk, R., \& Sage, K. (2010). A comparative study of dexmedetomidine with midazolam and midazolam alone for sedation 
during elective awake fiberoptic intubation. Journal of clinical anesthesia,22(1), 35-40.

14. Hassan, N. E., Betz, B. W., Cole, M. R., Wincek, J., Reischman, D., Sanfilippo, D. J., ... \& Kopec, J. S. (2011). Randomized controlled trial for intermittent versus continuous propofol sedation for pediatric brain and spine magnetic resonance imaging studies. Pediatric Critical Care Medicine| Society of Critical Care Medicine,12(6), e262e265.

15. Candiotti, K. A., Bergese, S. D., Bokesch, P. M., Feldman, M. A., Wisemandle, W., Bekker, A. Y., \& MAC Study Group. (2010). Monitored anesthesia care with dexmedetomidine: a prospective, randomized, double-blind, multicenter trial. Anesthesia \& Analgesia, 110(1), 47-56.

16. Glass, P. S., Bloom, M., Kearse, L., Rosow, C., Sebel, P., \& Manberg, P. (1997). Bispectral analysis measures sedation and memory effects of propofol, midazolam, isoflurane, and alfentanil in healthy volunteers. The Journal of the American Society of Anesthesiologists, 86(4), 836-847.

17. Jalowiecki, P., Rudner, R., Gonciarz, M., Kawecki, P., Petelenz, M., \& Dziurdzik, P. (2005). Sole use of dexmedetomidine has limited utility for conscious sedation during outpatient colonoscopy. The Journal of the American Society of Anesthesiologists, 103(2), 269-273.

18. Kamibayashi, T., Maze, M., Weiskopf, R. B., Weiskopf, R. B., \& Todd, M. M. (2000). Clinical uses of $\alpha 2$-adrenergic agonists. The Journal of the American Society of Anesthesiologists, 93(5), 13451349.

19. Taittonen, M. T., Kirvelä, O. A., Aantaa, R., \& Kanto, J. H. (1997). Effect of clonidine and dexmedetomidine premedication on perioperative oxygen consumption and haemodynamic state. British journal of anaesthesia,78(4), 400406.

20. Deurenberg-Yap, M., Schmidt, G., van Staveren, W. A., \& Deurenberg, P. (2000). The paradox of low body mass index and high body fat percentage among Chinese, Malays and Indians in Singapore. International journal of obesity, 24(8), 1011-1017.

21. Venn, R. M., Hell, J., \& Grounds, R. M. (2000). Respiratory effects of dexmedetomidine in the surgical patient requiring intensive care. Critical Care, 4(5), 1-7.

22. Ebert, T. J., Muzi, M., Berens, R., Goff, D., \& Kampine, J. P. (1992). Sympathetic responses to induction of anesthesia in humans with propofol or etomidate. The Journal of the American Society of Anesthesiologists, 76(5), 725-733.

23. Ebert, T. J., Hall, J. E., Barney, J. A., Uhrich, T. D., \& Colinco, M. D. (2000). The effects of increasing plasma concentrations of dexmedetomidine in humans. The Journal of the American Society of Anesthesiologists, 93(2), 382-394.

24. Bloor, B. C., Ward, D. S., Belleville, J. P., \& Maze, M. (1992). Effects of intravenous dexmedetomidine in humans: II. Hemodynamic changes. The Journal of the American Society of Anesthesiologists, 77(6), 1134-1142.

25. Singh, H., Poluha, W., Cheang, M., Choptain, N., Inegbu, E., Baron, K., \& Taback, S. P. (2008). Propofol for sedation during colonoscopy. Cochrane database of systematic reviews, (4).

26. Aantaa, R. (1991). Assessment of the Sedative Effects of Dexmedetomidine, an a2-Adrenoceptor Agonist, with Analysis of Saccadic Eye Movements. Pharmacology \& toxicology, 68(5), 394-398.

Cite this article: Sabia Siddika et al (2021). Comparison between Propofol and Dexmedetomidine for Conscious Sedation in Patients Undergoing Outpatient Colonoscopy. EAS J Anesthesiol Crit Care, 3(2), 23-29. 